\title{
Three-dimensional few-circle optical pulses in the inhomogeneous environment of carbon nanotubes in an optical resonator
}

\author{
Ilya Dvuzhilov ${ }^{1, *}$, Yulia Dvuzhilova ${ }^{2}$, Mikhail Belonenko ${ }^{1,2}$, Irina Zaporotskova ${ }^{1}$, and \\ Natalia Boroznina ${ }^{1}$ \\ ${ }^{1}$ Volgograd State University, Volgograd 400062, Russia \\ ${ }^{2}$ Volgograd Institute of Business, Volgograd 400010, Russia
}

\begin{abstract}
We consider the task about few-circle optical pulses dynamics (light bullets) in the inhomogeneous environment of carbon nanotubes in a resonant optical cavity. Our calculations show that the optical resonator can create conditions, which can provide monitoring and controlling the shape and the velocity of the optical pulse. We show that the propagation of the light bullets is stable.
\end{abstract}

\section{Introduction}

Carbon nanotubes (CNTs) because of their nonlinear optical properties have been used as generator of a media with unique features. CNTs have height interest in the research community owing to the simplicity of their structure and their unique properties, which in turn contributed significantly to both the development in optical pulses propagation studies, as well as the development of optical devices based on them [1-2]. Ability to use CNTs as a medium for the formation of light bullets is the one of the most important feature.

Usually the propagation of optical pulses is considered in a uniform CNT environment that does not allow one to control the pulse velocity. However, if the propagation velocity of light bullets is determined by the refractive index of the medium and can vary within a wide range, one can perform a further modulation in the refractive index. In turn, this favours the formation of media with a modulated refractive index, thereby enabling the control of the propagation velocity of light bullets as well as the delay time.

\section{Basic equations}

The electromagnetic pulse propagation in carbon nanotube with optical resonator array has been considered, wherein both the pulse electric field and the current are directed along the nanotube axis.

For carbon nanotubes of the zigzag type, namely $(m, 0)$, the dispersion relation for the energy of conduction electrons reads [3]:

" Corresponding author: dvuzhilov.ilya@,volsu.ru 


$$
\varepsilon_{S}(\boldsymbol{p})= \pm \gamma \sqrt{1+4 \cos (a \boldsymbol{p}) \cos (\pi \mathrm{s} / m)+4 \cos ^{2}(\pi \mathrm{s} / m)}
$$

where $s=1,2 \ldots m$, nanotube has a type $(m, 0), \gamma \approx 2.7 \mathrm{eV}, a=3 b / 2 \hbar, b=0.142 \mathrm{~nm}-$ is a distance between neighboring carbon atoms.

Maxwell equations for non-magnetic dielectric environment bring to view:

$$
\frac{\partial^{2} \boldsymbol{E}}{\partial z^{2}}+\frac{\partial^{2} \boldsymbol{E}}{\partial r^{2}}-\frac{1}{\mathrm{c}^{2}} \frac{\partial^{2} \boldsymbol{E}}{\partial \mathrm{t}^{2}}+\frac{4 \pi}{\mathrm{c}} \frac{\partial j}{\partial \mathrm{t}}+\frac{4 \pi}{\mathrm{c}^{2}} \frac{\partial^{2} P}{\partial \mathrm{t}^{2}}=0,
$$

where $\mathbf{E}$ - is the electric field of the light wave, $j$ - is the electron current density, $t$ - is the time, and $c$ - is the light velocity in the medium., $P=\alpha E$.

Solving the problem, we neglect the diffraction spreading of the laser beam in the direction along the CNT axis. We ignore the substrate electric field as well. Note that since both the typical size of CNTs and the distance between them are much smaller than the spatial domain typical size in which a few-cycle pulse is localized, we can use the approximation of continuous medium and consider the current distributed over the volume.

The performed investigation allows us to make the following conclusions:

The three-dimensional few-cycle optical pulse (light bullet) propagation in inhomogeneous medium of carbon nanotubes in optical resonator is stable.

At large times the pulse remains localized along the axis of the cylindrical resonator.

The formation of a stable structure, which occurs due to the reflection of waves from the walls of the resonator and subsequent interference. Such an interaction makes it possible to reduce the width of the pulse in the direction of the transverse axis of the resonator.

\section{References}

[1] A.V. Zhukov, R.E. Bouffanais, M.B. Belonenko, N.N. Konobeeva, Yu.V. Nevzorova, T.F. George, Eur. Phys. J. D 69, 129 (2015)

[2] A.V. Zhukov, R.E. Bouffanais, G. Fedorov, M.B. Belonenko, J. Appl. Phys. 114, 143106 (2013)

[3] A.V. Zhukov, R. Bouffanais, M.B. Belonenko, I.S. Dvuzhilov, Yu.V. Nevzorova, Appl. Phys. B 123, 196 (2017) 